\title{
Multiaxial cycle counting method for non- proportional multiaxial variable loading signals based on modified maximal shear stress
}

\author{
Matus Margetin ${ }^{1}$ and Dominik Biro ${ }^{1}$ \\ ${ }^{1}$ Slovak University of Technology, Faculty of Mechanical Engineering, Namestie slobody 17, 81231 \\ Bratislava, Slovakia
}

\begin{abstract}
One of the most challenging task in field of multiaxial fatigue is fatigue lifetime estimation of components loaded with multiaxial nonproportional variable amplitude loading. While this task consists of multiple smaller problems, one of the most crucial ones is loading cycles identification (and extraction) for future use with multiaxial damage criterions. By now, several cycle counting methods have been proposed for multiaxial loading conditions. The most wildly accepted methods are Bannantine-Socie's method and Wang-Brown's method (which has been later modified by Meggiolaro and Castro). The aim of this paper is the comparison of newly developed method with Bannantine-Socie's method and Wang-Brown's method. The new cycle counting method is based on cycle identification in relative maximum shear stress histories (calculated from multiaxial loading histories). The extracted data than composes part of each loading channel of multiaxial loading histories corresponding to identified loading cycle. The comparison of chosen methods has been done by using data sets created by authors as well as using real measured data from real operation.
\end{abstract}

\section{Introduction}

While multiaxial fatigue lifetime estimation is by today still very actual topic in field of material fatigue research, few articles can be found dealing with problem of multiaxial cycle counting methods. And yet correct cycle identification is key factor in successful process of fatigue lifetime estimation. By today multiple multiaxial cycle counting techniques have been proposed, but due to complexity of fatigue lifetime estimation under multiaxial variable nonproportional loading conditions, the majority of cycle counting method has very strict rules of usage and can be use universally (usually they are connected to specific multiaxial damage criterion) [1,2]. As far authors know only two widely accepted universal methods have been proposed by today. The aim of this article is to compare these two methods with cycle counting procedure proposed by authors based on cycle identification in relative maximum shear stress histories (calculated from multiaxial loading histories). 


\section{Methods for cycle identification in multiaxial loading signals}

For the purpose of this article three cycle counting methods will be discussed, two of them are well known and established methods, and one method is developed and prepared by authors.

\subsection{Bannantine-Socie method}

One of the first systematic attempt to solve the problem of multiaxial variable amplitude cycle identification for purpose of fatigue lifetime estimation was done by Bannantine and Socie [3]. They proposed to use standard rainflow algorithm which has to be tuned to capture some additional information. The multiaxial loading signal acting in critical plane has to be recalculated into the multiple plane corresponding to different coordinate systems. Then based on the multiaxial fatigue damage criterion used for fatigue estimation the normal or shear stress (strain) loading histories in each plane is processed using rainflow algorithm. Simultaneously the modified rainflow algorithm store needed information of events occurring in second stress (strain) signal for each extracted cycle (in our case rainflow algorithm extract cycles from shear signal and take additional information from normal stress signal see Fig. 1).

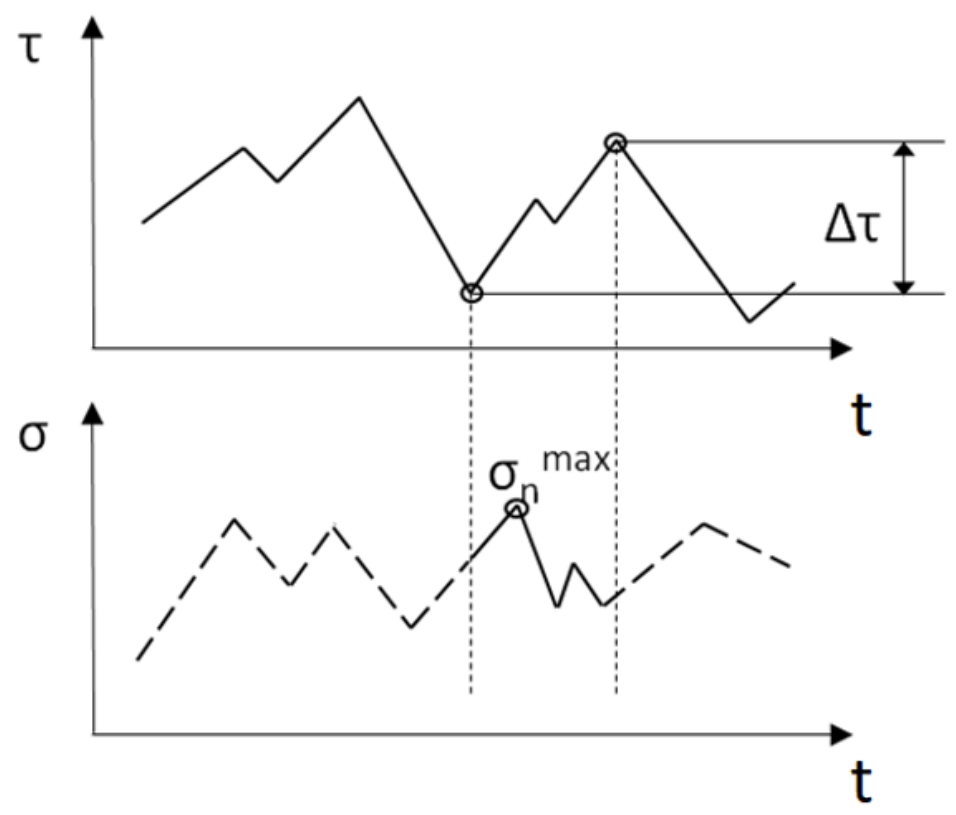

Fig. 1. Principle of modified rainflow algorithm proposed by Bannantine and Socie.

This method works very well with critical plane multiaxial models, as the stress signal is transformed into multiple plane before extraction. Therefore, extracted cycle could be used directly for damage calculation in corresponding plane. However, due to the fact that extraction has to run in each plane, the method could be very time consuming in case of long stress histories. 


\subsection{Wang-Brown method}

The cycle counting method based on different principles has been proposed by Wang and Brown $[4,5]$. They developed method based on cycle identification in equivalent stress (strain) space. Modified Von Mises stress (strain) signal calculated from real loading signals is used as an equivalent signal. Modification is based on the principle that real normal and shear signals have to be shifted to prevent loss of stress (strain) orientation (typical example is $90^{\circ}$ out-of-phase loading signal where Von Mises stress remains constant) leading to underestimating of loading conditions. The normal and shear stress (strain) signals are then rearranged in the way that they start in position where modified Von Mises stress is minimal (Fig. 2).

a)

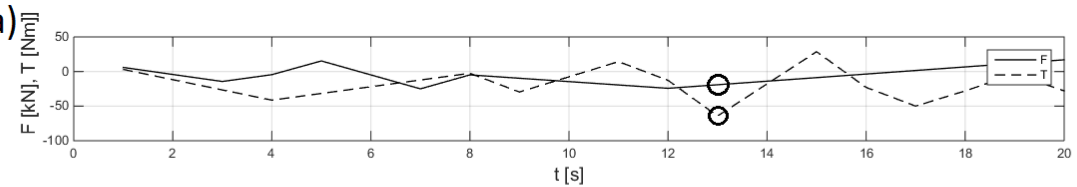

b)
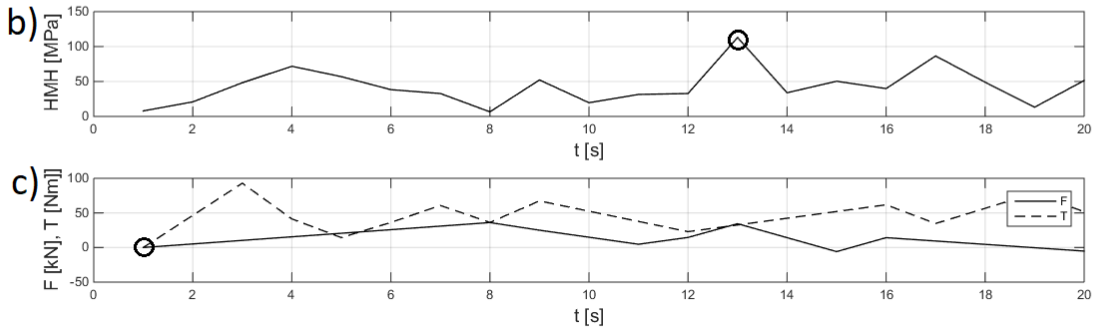

Fig. 2. a) measured signal, b) Mises stress calculated from measured signal, c) rearranged signal used for Wang-Brown procedure.

Then starting in initial point and ending in point with maximal value one half cycle is identified based on the principle shown in Fig. 3. The remaining parts of the signal (dashed line) that have to be processed using same procedure to obtain another half cycle.

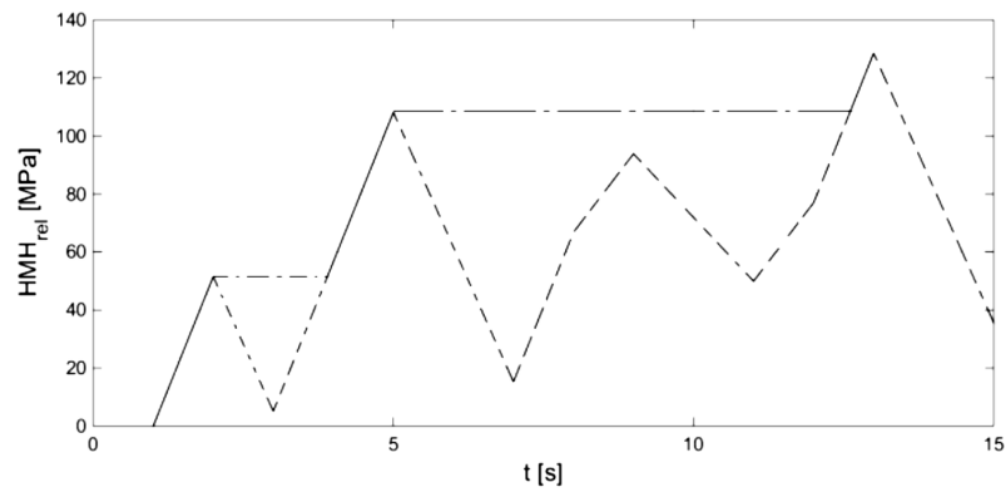


Fig. 3. Wang-Brown half cycle identification

Unlike the Bannantine-Socie method the Wang-Brown algorithm search through the loading signal only once to identify loading cycles. The extracted cycles than can be used based on multiaxial damage criterion. However, the method only extracts half-loading cycles which is one big disadvantage against Bannantine-Socie method.

\subsection{Newly proposed method}

Both mentioned methods have some disadvantages. Third method, based on proposition by authors of this paper, was used. Like Wang-Brown method the basic principle of proposed counting procedure, is to calculate the equivalent stress which is used for cycle identification. The maximal shear stress value is used as equivalent stress signal as authors believe that reversible shear stress is driven force of fatigue damaging mechanism. The fully enclosed cycles are then identified using modified rainflow algorithm. Start and end point of each identified cycle is then used to reconstruction of loading cycle from original measured data. Cycle extraction procedure is shown in Fig. 4.

a)

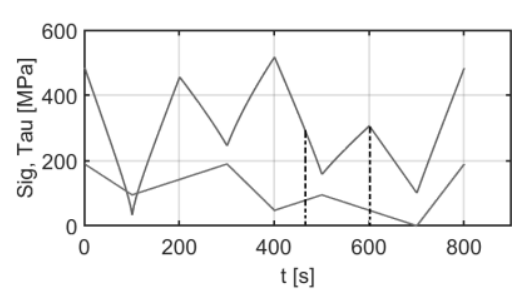

b)

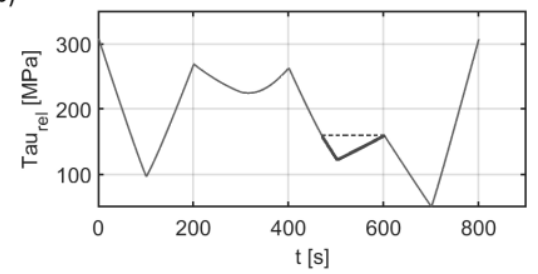

c)

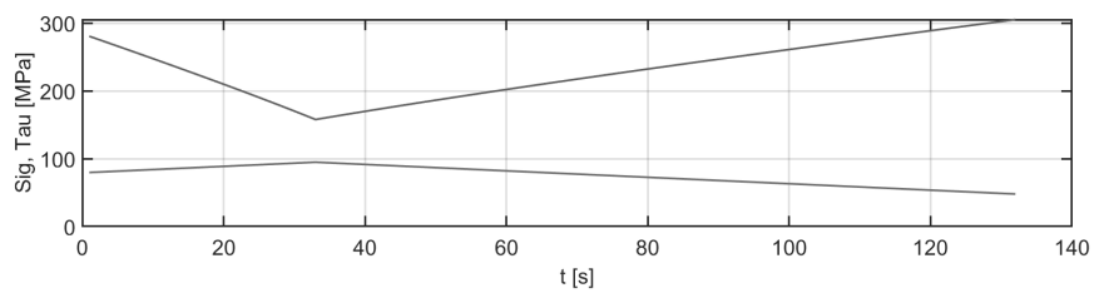

Fig. 4. Principle of proposed method: a) measured signal (dashed lines show the start and end point of extracted cycle), b) Relative maximal shear with identified cycle, c) extracted cycle.

Proposed procedure combines the benefits of both previously mentioned methods. As the extracting procedure searches for the cycles only once, and still the output from the procedure are fully enclosed cycles.

\section{Multiaxial loading histories}

Two sets of multiaxial loading histories were used to compare aforementioned methods. The short model signals were used for comparing the sets of extracted cycles. The real operational loading was used to compare the overall performance of all three methods in fatigue lifetime estimation.

\subsection{Model loading histories}


Short stress loading histories similar to model loading histories from Wang and Brown article $[4,5]$ were used for cycle extraction comparison. As Bannantine-Socie method works on different principles as other two methods, extracted cycles for $30^{\circ}$ plane orientation were used. The model loading signals in form of time functions as well as in form of loading path can be seen in Fig. 5 .
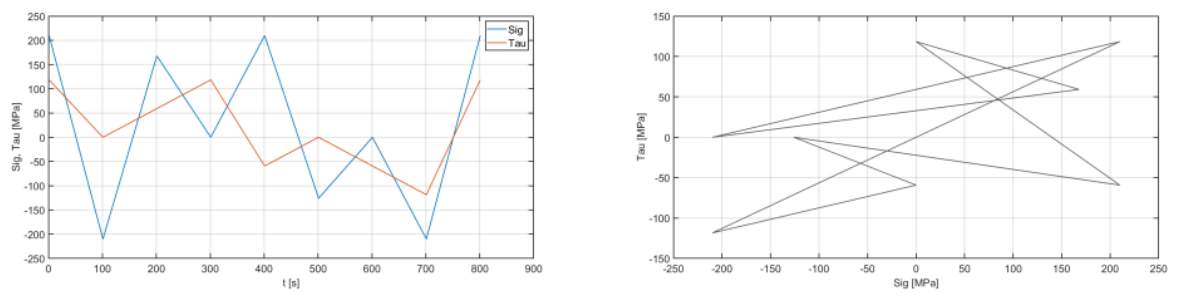

Fig. 5. Model loading signals.

\subsection{Real operational loading histories}

Second loading signals chosen for the method comparison, was real operational loading measured on trailer axle during durability tests. The methodology of measurement was described in [6,7]. The loading signal in time domain as well as in form of loading path is shown in Fig. 6. In case of real operational loading it is impossible to directly compare extracted cycles. Instead, we chose to compare finite fatigue lifetime estimation calculated from cycle sets obtained by all three methods. The results of fatigue lifetime estimation of component under operational loadings is strongly depended on chosen calculation methods. Following approaches have been used in conjunction with aforementioned cycle extracting methods. Findley [8] multiaxial fatigue criterion (eq. 2) was used to calculate equivalent stress amplitudes in each plane. As the extracted shear stress amplitudes have nonzero mean stress value, Wang and Miller [9] correction model (eq. 1) was used. The model recalculate extracted shear stress amplitude with mean value and extracted mean value $\left(\tau_{a m}\right.$ , $\left.\tau_{m}\right)$ to equivalent shear stress amplitude $\tau_{a}$ which can be than used in multiaxial fatigue criterion.

$$
\begin{gathered}
\tau_{a}=\tau_{a m} \cdot e^{\tau_{m} / \tau_{f}} \\
\tau_{f i n}=\left(\tau_{a}+k \cdot \sigma_{n}\right)_{\max }=\tau_{f}^{*}(2 N f)
\end{gathered}
$$

Total damage in each plane used to fatigue lifetime estimation has been calculated using Palmgren-Miner [10] linear damage accumulation rule (3).

$$
D=\Sigma\left(n_{i} / N_{f i}\right)
$$
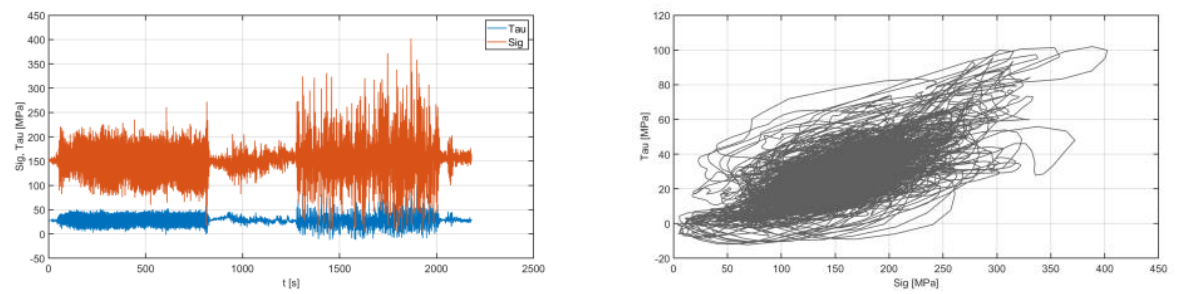

Fig. 6. Signal measured on trailer axel during durability tests. 


\section{Results}

\subsection{Model loading histories}

The extracted cycles for all three methods are shown in table 1. As it can be seen the major cycle was correctly identified by all three methods. The main difference between all three methods is the number of identified minor cycles. As Wang-Brown method in comparison to other methods extract only half cycles, the number of extracted cycle is much bigger (the biggest cycle is extracted twice) which can lead to very conservative results.

Table 1. Set of extracted cycles (half cycles) for $30^{\circ}$ plane orientation.

\begin{tabular}{|c|c|c|c|c|c|}
\hline \multicolumn{2}{|c|}{ Wang-Brown } & \multicolumn{2}{c|}{ Bannantine-Socie } & \multicolumn{2}{c|}{ Margetin-Biro } \\
\hline $\mathrm{Ta}[\mathrm{MPa}]$ & $\mathrm{Sn}[\mathrm{MPa}]$ & $\mathrm{Ta}[\mathrm{MPa}]$ & $\mathrm{Sn}[\mathrm{MPa}]$ & $\mathrm{Ta}[\mathrm{MPa}]$ & $\mathrm{Sn}[\mathrm{MPa}]$ \\
\hline 119 & 210 & 59 & 168 & 46 & 109 \\
\hline 59 & 210 & 30 & 210 & 20 & 210 \\
\hline 30 & 168 & 119 & 210 & 28 & -6 \\
\hline 76 & 179 & - & - & 98 & 208 \\
\hline 30 & 210 & - & - & 119 & 210 \\
\hline 30 & 0 & - & - & - & - \\
\hline 14 & 0 & - & - & - & - \\
\hline 119 & 210 & - & - & - & \\
\hline
\end{tabular}

\subsection{Real operational loading histories}

After cycle extraction, fatigue lifetime was estimated for all three methods. As Findley multiaxial criterion was used, fatigue lifetime is determined by accumulated damage in critical plane. Extracted cycles in form of histogram matrix for critical plane corresponding to all three cycle counting techniques can be seen in Fig. 7.
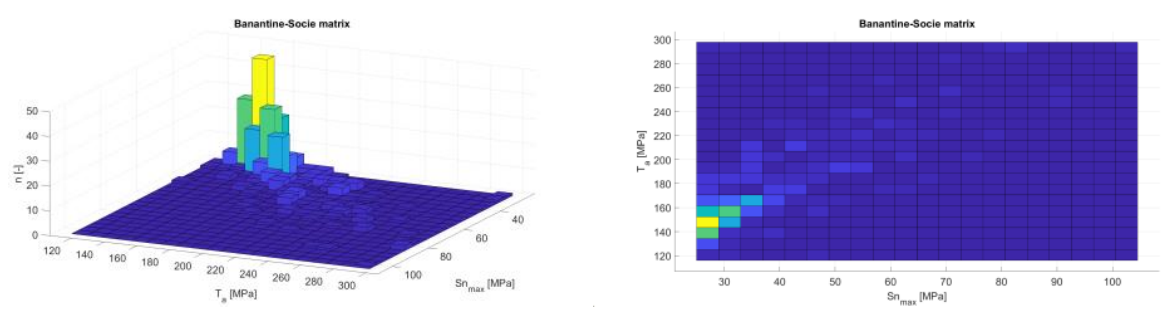

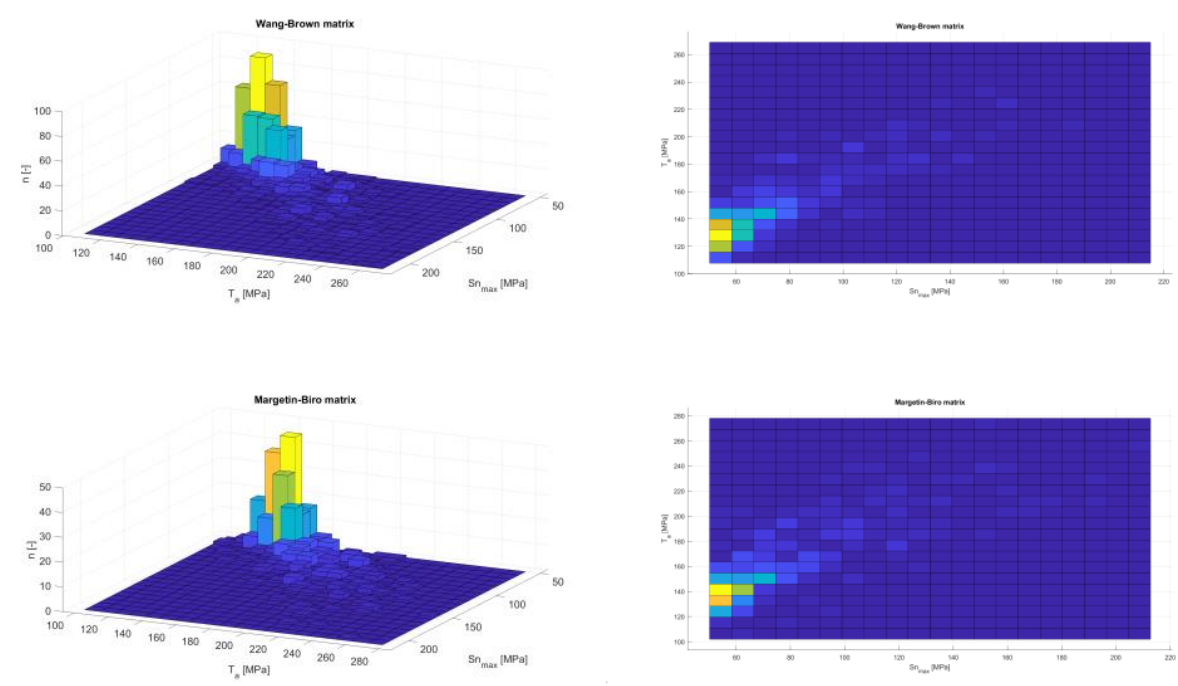

Fig. 7. Matrix with extracted cycles in critical plane.

Estimated fatigue lifetimes $\left(\mathrm{N}_{\mathrm{f}}\right)$, orientations of critical plane $\left(\Phi_{\text {cri }}\right)$ as well as predicted lifetime ratios to Wang-Brown prediction $\left(\mathrm{R}_{\mathrm{WB}}\right)$ can be seen in table 2. Function between plane orientation (angle in Mohr circle) and fatigue lifetime for all three methods can be seen in Fig. 8. The critical plane with lowest number of cycles to failure is marked with circle.

Table 2. Calculated lifetime in number of block repetitions.

\begin{tabular}{|c|c|c|c|}
\hline & Bannantine-Socie & Margetin-Biro & Wang-Brown \\
\hline $\mathbf{N}_{\mathbf{f}}$ & 34504 & 152 & 63 \\
\hline $\boldsymbol{\Phi}_{\text {cri }}$ & $20.5^{\circ}$ & $24^{\circ}$ & $25.5^{\circ}$ \\
\hline $\mathbf{R}_{\mathbf{W B}}$ & 547.68 & 2.41 & 1 \\
\hline
\end{tabular}

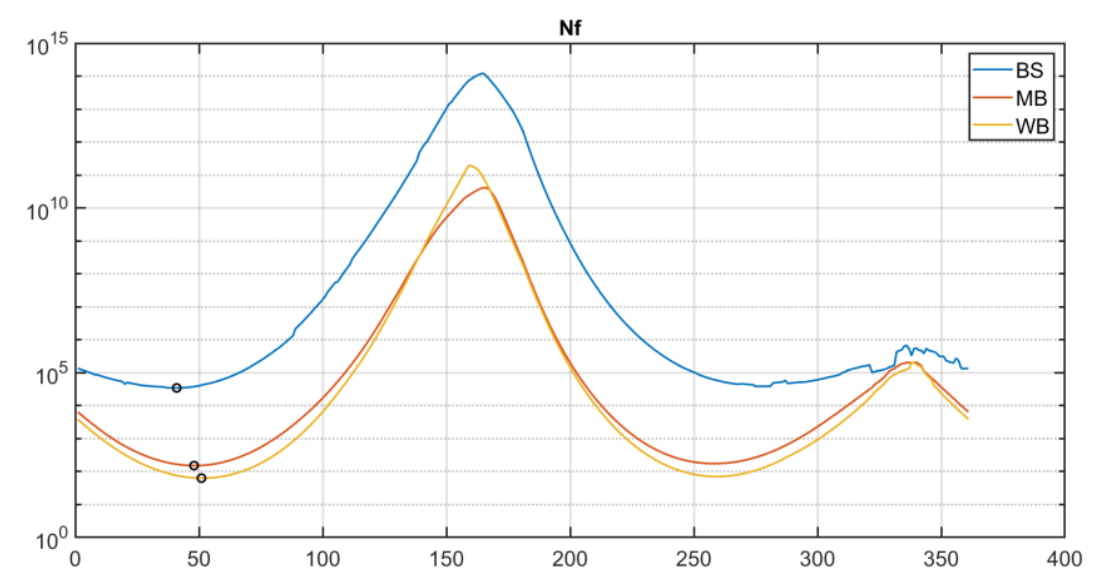

Fig. 8. Fatigue lifetime as a function of plane orientation. 


\section{Conclusion}

Three cycle counting methods for multiaxial variable amplitude loading were used to extract loading cycle from two sets of loading signals. Simple loading signal was used for comparison of extracted cycles. Real operational loading was used to demonstrate influence of cycle counting method on fatigue lifetime estimation. The following conclusion can be drawn:

- As Wang-Brown method extract only half cycles, the number of extracted cycles is much higher. This can lead to overconservative results.

- All three methods are capable to extract major cycles from the loading signals.

- When used with real operational loading Bannantine-Socie method extract cycles with lower maximal normal stresses which leads to much higher estimated lifetimes.

This work was supported by the Research \& Development Operational Programme funded by the ERDF ITMS: 26240220084 Science city Bratislava and by the Slovak Research and Development Agency under the contract No. APVV-17-0666.

\section{References}

1. V. Anes, L. Reis, B. Li, M. de Freitas, New cycle counting method for multiaxial fatigue, International Journal of Fatigue, 67, 78-94 (2014) https://doi.org/10.1016/j.ijfatigue.2014.02.010

2. X.-W. Wang, D.-G. Shang, Y.-J. Sun, X.-D. Liu, Algorithms for multiaxial cycle counting method and fatigue life prediction based on the weight function critical plane under random loading. International Journal of Damage Mechanics. (2019) https://doi.org/10.1177/1056789519831051

3. J.A. Bannantine, D.F. Socie, A variable amplitude multiaxial fatigue life prediction method, in: Fatigue under biaxial and multiaxial loading, European Structural Integrity Society ESIS Publication 10, Mechanical engineering publications, London, 1991, pp. 367-370

4. C.H. Wang, M.W. Brown, Life prediction techniques for variable amplitude multiaxial fatigue - Part 1: Theories, J Eng Meter Technol 14 (1996) 367-370

5. C.H. Wang, M.W. Brown, Life prediction techniques for variable amplitude multiaxial fatigue - Part 2: Comparison with experimental results, J Eng Meter Technol 118 (1996) 371-374

6. V. Chmelko, M. Garan, Long-term monitoring of strains in a real operation of structures. In: Proceedings of the 14th IMEKO TC10 Workshop on Technical Diagnostics 2016, Milano, Italy, p. 333-336.

7. V. Chmelko, V. Kliman, M. Garan, In-time monitoring of fatigue damage. Procedia Engineering 101, Elsevier 2015, p.93-100 https://doi.org/10.1016/j.proeng.2015.02.013

8. W.N. Findley, Fatigue of metals under combinations of stresses, Trans ASME 79 (1957) 1337-1338 5.D.L. McDiarmid, A general criterion for high cycle multiaxial fatigue failure, Fatigue Fract Eng M 14, 4 (1991) 429-453

9. C.H. Wang, K.J. Miller, The effect of mean shear stress on torsion fatigue behavior, Fract Engng Mater Struct 14 (1991) 293-307

10. Miner, M.A., Cumulative damage in fatigue, J. App. Mech., 12 (1945) A159-A164. 